\title{
Psychologische Leistungsdiagnostik
}

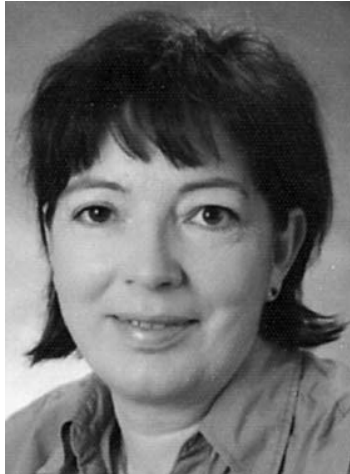

PD Dr. Monika Daseking

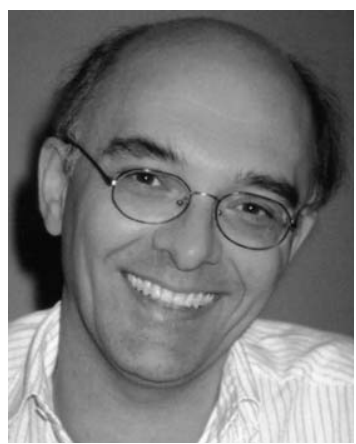

Prof. Dr. Franz Petermann

Bibliografie

DOI http://dx.doi.org/

10.1055/s-0033-1358680

Gesundheitswesen 2013;

75: 687-688

(c) Georg Thieme Verlag KG

Stuttgart · New York

ISSN 0941-3790

Korrespondenzadresse

PD Dr. Monika Daseking

Prof. Dr. Franz Petermann

Zentrum für Klinische

Psychologie und Rehabilitation

Universität Bremen

Grazer Straße 2 und 6

28359 Bremen

daseking@uni-bremen.de

fpeterm@uni-bremen.de
Der Begriff „Leistung“ ist aus der Alltagssprache nicht mehr wegzudenken. Ob in der Schule, im Sport, in der Ausbildung oder am Arbeitsplatz Leistung wird überall gefordert und soll optimiert werden. Aber auch die Leistungsmessung ist mit sehr unterschiedlichen Arbeits- und Lebensbereichen assoziiert: sei es die Überprüfung von Schul- oder Ausbildungsleistung durch Klausuren, die Leistungszulage im Beruf oder auch die Feststellung der Leistungsfähigkeit im Rahmen von Begutachtung oder Rehabilitation.

Die psychologische Leistungsdiagnostik weist eine mehr als 100 Jahre alte Tradition auf. Die Leistungsdiagnostik stellt dabei eine allgemeine Verfahrensklasse dar, die von der Messbarkeit der jeweiligen Fähigkeit ausgeht. Aufmerksamkeit und Konzentrationsfähigkeit oder Gedächtnisleistung gelten als klassische Domänen der Leistungsdiagnostik (z.B. [1]). Von Beginn an stellte aber auch die Intelligenzdiagnostik einen zentralen Aspekt der Leistungsmessung dar. $\mathrm{Zu}$ den überprüfbaren kognitiven Fähigkeiten zählen hier unter anderem sprachbezogene Fähigkeiten (z. B. Wortschatz, Sprachverständnis, Wissen im Sinne der kristallinen Intelligenz), visuellbasierte kognitive Fähigkeiten (Objekt- und Raumwahrnehmung, visuelle Analyse, räumlichkonstruktive Leistungen), visuelle und auditive Merk- und Lernfähigkeit, kognitive Verarbeitungsgeschwindigkeit, logisch-abstraktes Denken (fluide Intelligenz) und Aufmerksamkeit [2,3].

Psychologische Leistungsdiagnostik erfolgt über den Einsatz psychologischer Tests. Dabei handelt es sich nach Guthke ([4], S. 108) um ein „wissenschaftlich entwickeltes und überprüftes Routineverfahren, bei dem in standardisierten Situationen Verhalten - provoziert durch definierte Anforderungen - registriert bzw. Verhaltensmerkmale von Personen oder Personengruppen erfasst werden, die als Indikatoren für bestimmte Eigenschaften, Zustände oder Beziehungen dienen sollen“. Auf der Basis von Tests lassen sich quantitative Aussagen treffen; es kann also bestimmt werden, welchen relativen Grad an Merkmalsausprägung ein Individuum innerhalb einer Gruppe oder bezogen auf ein Kriterium aufweist. Über die Darstellung eines normbasierten und ressourcenorientierten Leistungsprofils ermöglicht psychologische Leistungsdiagnostik mittels psychometrischer Tests die Feststellung der kognitiven Fähigkeit einer Person im Sinne von Leistungsstärken und -schwächen [5,6].

Für solche psychometrischen Verfahren finden sich sehr unterschiedliche Systematisierungsvorschläge. Eine Systematisierung unter inhaltlichen
Aspekten fasst in der Rubrik Leistungstests Verfahren wie Entwicklungs-, Intelligenz-, Schuloder spezielle Funktionsprüfungs- und Eignungstests zusammen (vgl. dazu [7]). Testverfahren, die im Bereich der Leistungsdiagnostik zum Einsatz kommen, können dabei als Papier-und-Bleistift-Tests oder als computergestützte Verfahren konzipiert sein. Ein Unterscheidungsmerkmal betrifft die Art der Lösung: sind Antworten in einem freien Format erforderlich oder kann die Lösung aus vorgegebenen Antwortalternativen ausgewählt werden (multiple choice). Dabei spielt es weiterhin eine Rolle, ob die Schnelligkeit (speed) oder die Richtigkeit der Aufgabenbearbeitung (power) zur Bewertung herangezogen werden.

Die einmalige Bestimmung eines Leistungs- oder Fähigkeitsniveaus reicht zunächst aus, um eine definierte Eigenschaft mittels eines entsprechenden diagnostischen Verfahrens, in der Regel also durch den Einsatz eines Tests, zu erfassen und damit die diagnostische Fragestellung zu beantworten („Ist die Person hochbegabt?“, „Hat dieses Kind eine Lese-Rechtschreibstörung?“, „Welche Schulform soll mein Kind besuchen?"). Allerdings ist es oft mit einer einfachen Antwort nicht getan, in vielen Fällen begründet die Befundermittlung Förder- und Therapiemaßnahmen, die wiederum evaluiert werden müssen. Selbstverständlich können kontinuierlich erhobene Daten aus der Leistungsdiagnostik den Verlauf und Erfolg solcher Maßnahmen bewerten und steuern, sofern sie kleinschrittig erhoben werden. Gerade am Beispiel der Lese-Rechtschreibstörung kann deutlich gemacht werden, dass Leistungsdiagnostik nicht nur stabile Eigenschaften erfassen, sondern auch Veränderung über die Zeit abbilden kann (hier Verbesserung der Rechtschreibleistung als Therapieerfolg [8]).

In der aktuellen Diskussion sind die Begriffe „Kompetenz“ und „Kompetenzdiagnostik“ neben den Leistungsbegriff getreten, die vor allem im pädagogischen Kontext ihren Platz gefunden haben (z.B. mathematische Kompetenz, Lesekompetenz, Problemlösekompetenz) und stärker auf eine Beschreibung von Leistungsdispositionen abzielen. Hier wird Leistung aus der anwendungsorientierten Perspektive betrachtet. Auch Kompetenzdiagnostik erfolgt durch den Einsatz standardisierter (Leistungs-)Tests. Kompetenzen - wie die Lesekompetenz - werden erworben, dem Erwerb dieser Fertigkeiten liegen jedoch andere Teilleistungen und Funktionen zugrunde, die als Vorläuferfähigkeiten bezeichnet werden. In diesem Zusammenhang spielt die schulärztliche 
Einschulungsuntersuchung eine besondere Rolle. Im Rahmen der Feststellung des kognitiven Entwicklungsstands wie beispielsweise durch das Screening SOPESS [9] lassen sich Förderbedarfe erheben. Durch daraus abgeleitete Maßnahmen können Kinder bei einer gesunden schulischen Entwicklung unterstützt werden [10]. Wie Vorläuferfähigkeiten und Kompetenzerwerb in der Schule zusammenspielen, konnte in einer umfangreichen Studie zur prognostischen Validität von SOPESS aufgezeigt werden [11,12]. Dieser Zusammenhang konnte aber bereits auch für andere Tests zu Vorläuferfähigkeiten nachgewiesen werden [13].

Auch im sozialmedizinischen Kontext kann die Leistungsdiagnostik wichtige Informationen im Rahmen der Entscheidungsfindung liefern. Beginnend mit der Fragestellung der Schuleingangsdiagnostik lässt sich der Bogen hier über die Feststellung von Förderbedarf bei Teilleistungsschwächen oder -störungen im schulischen Kontext (wie Lese-Rechtschreibstörung, Dyskalkulie) bis hin zur sozialmedizinischen Begutachtung von Arbeitsfähigkeit oder Rehabilitationsbedarf sowie der Empfehlung zu beruflichen Wiedereingliederungsmaßnahmen nach somatischen, psychischen oder neurologischen Erkrankungen spannen. Leistungsdiagnostik kann hier wichtige Ressourcen aufdecken, die den Verlauf von Maßnahmen positiv unterstützen, gleichzeitig aber auch dazu beitragen, behandlungs- oder förderbedürftige Schwächen und Hilfebedarf zu identifizieren.

Im vorliegenden Heft werden die klassischen Domänen der Leistungsdiagnostik aufgegriffen: Intelligenz, Aufmerksamkeit und Gedächtnis. Pauls, Lepach und Petermann [14] zeigen in ihrem Beitrag auf, in welcher Weise die differenzierte Diagnostik von Gedächtnisleistungen bei Patienten mit Depression dazu beitragen kann, die depressive Störung von einer dementiellen Erkrankung abzugrenzen. Gleichzeitig lassen sich aus dem Profil neuropsychologischer Leistungen aber auch Behandlungsmaßnahmen effektiv ableiten und strukturieren.

In eine ähnliche Richtung weist der Beitrag von Stemmler und Kollegen [15] der sich mit dem Zusammenhang von Gedächtnis und Intelligenz auseinandersetzt. Ausgehend von der Feststellung, dass aufgrund der steigenden Lebenserwartung die Anzahl von Personen zunimmt, die im höheren Lebensalter an einer Demenz erkranken [16], stellt die Suche nach zuverlässigen Früherkennungsmethoden und effektiven Behandlungsmöglichkeiten eine große Herausforderung dar. Inwieweit ein Syndrom-Kurztest (SKT) zur Gedächtnisdiagnostik hier wichtige Informationen liefern kann, stellen Stemmler et al. [15] zur Diskussion. Vor allem der Vergleich mit der Entwicklung von Intelligenzleistungen im höheren Lebensalter zeigt, dass nicht jede Leistungsabnahme mit einem dementiellen Abbauprozess gleichzusetzen ist, sondern dass es hier notwendig ist, zwischen normalen und auffälligen Veränderungen der kognitiven Leistungsfähigkeit zu unterscheiden.

Zur Erklärung der Zusammenhänge zwischen Intelligenz und Gedächtnis trägt auch der Beitrag von Lepach und Kollegen [17] bei. Es zeigt sich hier noch einmal, dass die Zusammenhänge zwischen beiden Leistungsbereichen nur moderat ausfallen. Durch die Gedächtnisleistungen, wie sie mit der Wechsler Memory Scale - IV (WMS-IV) erhoben werden können, lässt sich der Gesamt-Intelligenzwert der Wechsler Adult Intelligence Scale - IV (WAIS-IV) gut vorhersagen. Die umgekehrte Vorhersage vom IQ auf das Gedächtnis gelingt jedoch nur unzureichend. Daraus lässt sich schlussfolgern, dass zur differenzierten Leistungsbestimmung beide Verfahren eingesetzt werden sollten, da sie sich gegenseitig in ihrer Aussage ergänzen und nicht ersetzen.

Auch Theiling und Kollegen [18] zeigen in ihrem Beitrag zur ADHS im Erwachsenenalter, welche Rolle Leistungstests im Kontext einer ADHS-Diagnostik spielen. Über den Vergleich der Ergebnisse in einem Intelligenztest (WAIS-IV) mit Aussagen von Patienten zur Selbsteinschätzung der störungsspezifischen Symptomatik wird deutlich, dass Selbst- und Fremdratings die Störung zwar gut auf der Verhaltensebene abbilden können, sich daraus jedoch keine Aussagen über die kognitiven Defizite ableiten lassen, die im Einzelfall mit der Störung assoziiert sein können. Diese Einschränkungen in der Denkfähigkeit können jedoch den Lebensweg betroffener Personen deutlich beeinflussen. Auch hier lassen sich dann auf der Basis eines differenzierten Stärken- und Schwächenprofils gezielte Hinweise im Rahmen einer sozialmedizinischen Begutachtung und notwendige Therapiemaßnahmen ableiten.

Interessenkonflikt: Die Autoren geben an, dass kein Interessenkonflikt besteht.

\section{Literatur}

1 Hasselhorn M, Hartmann U. Lern-und Aufmerksamkeitsstörungen. Kindh Entwickl 2011; 20: 1-3

2 Daseking $M$, Janke N, Petermann F. Intelligenzdiagnostik. Monatsschr Kinderh 2006; 154: 314-319

3 Petermann F. Intelligenzdiagnostik. Kindh Entwickl 2006; 15: 71-75

4 Guthke J. Testtheorie (Testmodelle). In: Guthke J, Böttcher HR, Sprung L, Hrsg. Psychodiagnostik: ein Lehr- und Arbeitsbuch für Psychologen sowie empirisch arbeitende Humanwissenschaftler. Berlin: DVW; 1990; 105-200

5 Kastner J, Lipsius M, Hecking $M$ et al. Kognitive Leistungsprofile motorisch-und sprachentwicklungsverzögerter Vorschulkinder. Kindh Entwickl 2011; 20: 173-185

6 Jantzer V, Parzer P, Lehmkuhl U et al. Neuere Entwicklungen zur Diagnostik psychischer Störungen im Jugendalter. Kindh Entwickl 2012; 21: $198-207$

7 Brähler E, Holling H, Leutner D et al., Hrsg. Brickenkamp. Handbuch Psychologischer und Pädagogischer Tests. Göttingen: Hogrefe; 2002

8 Ise E, Engel RR, Schulte-Körne G. Was hilft bei der Lese-Rechtschreibstörung? Kindh Entwickl 2012; 21: 122-136

9 Petermann F, Oldenhage M, Simon K et al. Sozialpädiatrisches Entwicklungsscreening für Schuleingangsuntersuchungen (SOPESS). Bielefeld: LIGA.NRW; 2009

10 Daseking $M$, Petermann F, Röske $D$ et al. Entwicklung und Normierung des Einschulungsscreenings SOPESS. Gesundheitswesen 2009; 71: 648-655

11 Daseking $M$, Petermann $F$, Simon $K$ et al. Vorhersage von schulischen Lernstörungen durch SOPESS. Gesundheitswesen 2011; 73: 650-659

12 Daseking $M$, Petermann $F$. Der Einfluss von Vorläuferfähigkeiten auf die Rechtschreib-, Lese- und Rechenleistung in der Grundschule. Gesundheitswesen 2011; 73: 644-649

13 Knievel J, Petermann F, Daseking M. Welche Vorläuferdefizite weisen Kinder mit einer kombinierten Rechtschreib- und Rechenschwäche auf? Diagnostica 2011; 57: 212-224

14 Pauls F, Lepach AC, Petermann F. Depression und Gedächtnis: Gedächtnisleistungen im Vergleich zwischen Depressive und Gesunden. Gesundheitswesen 2013; 75: 754-760

15 Stemmler $M$, Petermann $F$, Daseking $M$ et al. Diagnostik und Verlauf von kognitiven Fähigkeiten bei älteren Menschen. Gesundheitswesen 2013; 75: 761-767

16 Ziegler $U$, Doblhammer G. Prävalenz und Inzidenz von Demenz in Deutschland - Eine Studie auf Basis von Daten der gesetzlichen Krankenversicherungen von 2002. Gesundheitswesen 2009; 71: 281-290

17 Lepach AC, Daseking M, Petermann F et al. Zusammenhange von Intelligenz- und Gedächtnisdiagnostik anhand von WAIS-IV und WMS-IV. Gesundheitswesen 2013; 75: 775-781

18 Theiling J, Petermann F, Daseking M. Zusammenhang zwischen selbsteingeschätzter und der ADMS-Symptomatik Leistungsfähigkeit in der WAIS-IV und WMS-IV. Gesundheitswesen 2013; 75: 768-774 